Portland State University

PDXScholar

\title{
First-Order Amplitude for General State-to-State Transitions in Hydrogen by Projectile Impact
}

Jack C. Straton

Portland State University, straton@pdx.edu

Follow this and additional works at: https://pdxscholar.library.pdx.edu/phy_fac

Part of the Atomic, Molecular and Optical Physics Commons, and the Statistical, Nonlinear, and Soft Matter Physics Commons

Let us know how access to this document benefits you.

\section{Citation Details}

Straton, Jack C. "First-order amplitude for general state-to-state transitions in hydrogen by projectile impact." Physical Review A 43.3 (1991): 1381. DOI: http://dx.doi.org/10.1103/PhysRevA.43.1381

This Article is brought to you for free and open access. It has been accepted for inclusion in Physics Faculty Publications and Presentations by an authorized administrator of PDXScholar. Please contact us if we can make this document more accessible: pdxscholar@pdx.edu. 


\title{
First-order amplitude for general state-to-state transitions in hydrogen by projectile impact
}

\author{
Jack C. Straton \\ Department of Physics, Cardwell Hall, Kansas State University, Manhattan, Kansas 66506-2601
}

(Received 11 June 1990)

\begin{abstract}
The closed analytic form for bound-state transitions due to projectile impact is found in the intermediate representation. The coordinate integral is obtained by evaluating the remaining two integrals in the general multicenter integral derived previously [J.C. Straton, Phys. Rev. A 41, $71(1990)$ ]. Evaluating the remaining time integral depends upon relating a sum of modified Bessel functions of the second kind $K_{N+1 / 2}(z)$ to a simple polynomial in $1 / z$. The results of Van Den Bos and De Heer [Physica 34, 333 (1967)] are shown to be missing a phase factor of $(-i)^{\left(\ell^{\prime}+\ell\right)}$.
\end{abstract}

\section{INTRODUCTION}

The amplitudes for excitation from the hydrogen ground state into the $n=2$ and 3 excited states that were found by Van Den Bos and De Heer ${ }^{1}$ have provided the basis for comparison in many experimental ${ }^{2-5}$ and theoretical ${ }^{6-8}$ studies. It would be helpful to have transition amplitudes into the $n=4$ manifold, for which Park et al. ${ }^{3}$ have experimental results, and higher. Likewise, as the experimental study of multiple excitation of many-electron atoms by projectile impact proceeds, ${ }^{9-11}$ a full catalog of transitions between the excited hydrogenic states will be required for calculations using configuration-interaction wave functions (weighted products of one-electron wave functions). McGuire et al. ${ }^{12}$ have contributed to this catalog by calculating the $2 \mathrm{~s} \rightarrow 2 \mathrm{p}$ transition amplitude, and Straton and McGuire ${ }^{13}$ have given the entire set of transitions within the $n=1$ and 2 manifolds, including $21 m \rightarrow 21 m^{\prime}$ transitions, in cases where there may be a change in the screened nuclear charge. Since the latter effort required some 250 pages of notes (125 for each of two independent methods), it may be expected that including just the full $n=3$ manifold would be an enormous undertaking.

The present paper presents the alternative of deriving the transition amplitude once, for all states $n \ell m \lambda$ (with $\left.\lambda=Z_{T} / a_{0}\right)$ to all states $n^{\prime} \ell^{\prime} m^{\prime} \lambda^{\prime}$. In Sec. II, the coordinate integral is evaluated by utilizing the author's results for the general multicenter integral. The time integration is performed in Sec. III by utilizing a relation involving sums of modified Bessel functions of the second kind $K_{N+1 / 2}(z)$ to a polynomial in $1 / z$. The proof of this relation is given in the Appendix.

\section{SPATIAL INTEGRATION}

The first-order one-electron transition amplitude in intermediate representation is

$$
\begin{aligned}
a_{n^{\prime} \ell^{\prime}-m^{\prime} \lambda^{\prime}, n \ell m \lambda}^{1} & (\omega, B) \\
= & -i \int_{-\infty}^{\infty} d t e^{i \omega t} V_{n^{\prime} \ell^{\prime}-m^{\prime}, n \ell m}^{\lambda^{\prime}}(R),
\end{aligned}
$$

where

$$
V_{n^{\prime} \ell^{\prime}-m^{\prime}, n \ell m}^{\lambda}(R)=\int d^{3} r u_{n^{\prime} \ell^{\prime}-m^{\prime}}^{\lambda^{\prime}}(\mathbf{r}) V(\mathbf{R}, \mathbf{r}) u_{n \ell m}^{\lambda}(\mathbf{r})
$$

$\omega=E^{\prime}-E$,

and in the semiclassical approximation (SCA) the projectile of velocity $v$ follows a rectilinear path as a function of time,

$$
\mathbf{R}=B \hat{\mathbf{i}}+v t \hat{\mathbf{k}} .
$$

$B$ is the impact parameter.

The first-order SCA has been shown to be the intermediate representation equivalent of (the two-dimensional Fourier transform of) the plane-wave first Born approximation (FBA) of Schrödinger representation. ${ }^{14,15}$ This consideration dictates the choice of asymptotic Hamiltonian $H_{P T}$ and thus the interaction potential $V(\mathbf{R}, \mathbf{r})$ in (2). In initial-state Jacobi coordinates, ${ }^{16}$ the three-body Hamiltonian reduces to a Hamiltonian for two fictitious bodies when the center-of-mass momentum is set to zero,

$$
H=H_{P T}+H_{0}+V_{i},
$$

where the asymptotic translational Hamiltonian, of which plane waves are eigenstates when $Z_{T}=-Z_{e}$, is

$$
H_{P T}=\frac{-\nabla_{R}^{2}}{2 M}+\frac{Z_{p}\left(Z_{T}+Z_{e}\right)}{R},
$$

in which $M=m_{P}\left(m_{T}+m_{e}\right) /\left(m_{P}+m_{T}+m_{e}\right)$ is the three-body reduced mass of the fictitious projectile. The atomic Hamiltonian is

$$
H_{0}=\frac{-\nabla_{r}^{2}}{2 m}+\frac{Z_{T} Z_{e}}{r}
$$


in which $m=m_{T} m_{e} /\left(m_{T}+m_{e}\right)$ is the reduced mass of the fictitious electron, and the interaction potential is

$$
\begin{aligned}
V_{i}=V(\mathbf{R}, \mathbf{r})= & Z_{p} Z_{e}\left(\frac{1}{\left|\mathbf{R}-\left(m / m_{e}\right) \mathbf{r}\right|}-\frac{1}{R}\right) \\
& +Z_{p} Z_{T}\left(\frac{1}{\left|\mathbf{R}+\left(m / m_{T}\right) \mathbf{r}\right|}-\frac{1}{R}\right) .
\end{aligned}
$$

Atomic units $\left(\hbar=e=m_{\text {electron }}=1\right)$ are used throughout. If the bound particle is an electron $m_{e}=m_{\text {electron }}=$ 1 , then $m \simeq 1$. Thus $1 /\left|\mathbf{R}+\left(m / m_{T}\right) \mathbf{r}\right| \simeq 1 / R$, and the internuclear term is canceled to one part in a thousand, consistent with the rectilinear SCA approximation (4).

For generality, however, the present calculation will be of the quantity

$$
\mathcal{A}_{n^{\prime} \ell^{\prime}-m^{\prime}, n \ell m}^{\lambda}(\omega, B, \nu, Z)=\int_{-\infty}^{\infty} d t e^{i \omega t} \int d^{3} r u_{n^{\prime} \ell^{\prime}-m^{\prime}}^{\lambda^{\prime} *}(\mathbf{r}) Z_{p} Z\left(\frac{1}{|\mathbf{R}-\nu \mathbf{r}|}-\frac{1}{R}\right) u_{n \ell m(\mathbf{r})}
$$

so that the exact relation

$$
\begin{aligned}
a_{f i}^{1}(\omega, B)= & (-i) \mathcal{A}_{f i}\left(\omega, B, \frac{m}{m_{e}},-1\right) \\
& +(-i) \mathcal{A}_{f i}\left(\omega, B,-\frac{m}{m_{T}}, Z_{T}\right)
\end{aligned}
$$

may be used when desired.
The reduced form for the coordinate-space integral has been given by the author ${ }^{17}$ as a two-dimensional integral. In Sec. IV of that paper, the final two integrals are evaluated in a special case, giving the $1 /|\mathbf{R}-\mathbf{r}|$ term of the transition amplitude for $2,1, m \rightarrow 2,1,-m$. The following closely parallels that reduction. Equations $(n)$ from that paper will be referred to herein as $(n-\mathrm{I})$.

The amplitude (9) may be written as

$$
\mathcal{A}_{n^{\prime} \ell^{\prime}-m^{\prime} ; n \ell m}^{\lambda^{\prime}} \underset{\lambda}{\lambda}(\omega, B, \nu, Z)=\int_{-\infty}^{\infty} d t e^{i \omega t}(-1)^{-m^{\prime}} Z_{p} Z\left[\frac{1}{\nu} S_{n^{\prime} \ell^{\prime} m^{\prime} \lambda^{\prime} ; n \ell m \lambda ; \mathbb{1}}^{01,00}\left(0 ; 0, \frac{\mathbf{R}}{\nu}\right)-\frac{1}{R} S_{n^{\prime} \ell^{\prime} m^{\prime} \lambda^{\prime}, n \ell m \lambda}^{01}(0 ; 0)\right]
$$

where, from (31-I),

$$
S_{n^{\prime} \ell^{\prime} m^{\prime} \lambda^{\prime}, n \ell m \lambda ; \mathbb{1}}^{\eta_{1}, j_{1} ; \eta_{2} j_{2}}(0 ; 0, \mathbf{y})=\pi^{3 / 2} \int_{0}^{\infty} d \rho_{1} \int_{0}^{\infty} d \rho_{2} A_{n^{\prime} \ell^{\prime} m^{\prime} \lambda^{\prime}, n \ell m \lambda}^{\eta_{1} j_{1}}\left(\rho_{1} \mathbf{q}_{1}\right) A_{\mathbb{1}}^{\eta_{2} j_{2}} \quad\left(\rho_{2}, \mathbf{q}_{2}\right) \frac{e^{-\Omega / \Lambda}}{\Lambda^{3 / 2}} .
$$

In this expression

$A_{n^{\prime} \ell^{\prime} m^{\prime} \lambda^{\prime}, n \ell m \lambda}^{\eta_{1} j_{1}}\left(\rho_{1}, \mathbf{q}_{1}\right)$

$$
\begin{aligned}
& =\frac{e^{-\gamma^{2} / 4 \rho_{1}}}{2^{j_{1}} \sqrt{\pi}} \sum_{s^{\prime}=0}^{n^{\prime}-\ell^{\prime}-1} \frac{(-1)^{s^{\prime}}\left(\lambda^{\prime} / n^{\prime}\right)^{s^{\prime}+\ell^{\prime}} N_{n^{\prime} \ell^{\prime}} \lambda^{3 / 2}}{\left(n^{\prime}-\ell^{\prime}-1-s^{\prime}\right) !\left(2 \ell^{\prime}+1+s^{\prime}\right) ! s^{\prime} !} \\
& \times \sum_{s=0}^{n-\ell-1} \frac{(-1)^{s}(\lambda / n)^{s+\ell} N_{n \ell} \lambda^{3 / 2}}{(n-\ell-1-s) !(2 \ell+1+s) ! s !} \\
& \times \sum_{L=L_{\min }}^{\ell^{\prime}+\ell}(2)(-1)^{m^{\prime}+m}\left(\frac{\left(2 \ell^{\prime}+1\right)(2 \ell+1)(2 L+1)}{4 \pi}\right)^{1 / 2} \\
& \times\left(\begin{array}{ccc}
\ell^{\prime} & \ell & L \\
0 & 0 & 0
\end{array}\right)\left(\begin{array}{ccc}
\ell^{\prime} & \ell & L \\
m^{\prime} m & -m^{\prime}-m
\end{array}\right) \mathcal{Y}_{L, m^{\prime}+m}\left(\mathbf{q}_{1}\right) \frac{2^{L} H_{s^{\prime}+\ell^{\prime}+s+\ell-L+j_{1}}\left(\gamma / 2 \sqrt{\rho_{1}}\right)}{\rho_{1}^{\left(s^{\prime}+\ell^{\prime}+s+\ell-L+1+j_{1}\right) / 2}},
\end{aligned}
$$

in which

$$
\begin{aligned}
& \gamma=\frac{\lambda^{\prime}}{n^{\prime}}+\frac{\lambda}{n}+\eta_{1}, \\
& N_{n \ell}=\frac{2}{n^{2}} \sqrt{(n-\ell-1) !(n+\ell) !},
\end{aligned}
$$

$$
\begin{aligned}
& \mu=\max \left(\left|\ell^{\prime}-\ell\right|,\left|m^{\prime}+m\right|\right), \\
& L_{\min }=\left\{\begin{array}{l}
\mu \text { if } \ell^{\prime}+\ell+\mu \text { is even } \\
\mu+1 \text { if } \ell^{\prime}+\ell+\mu \text { is odd },
\end{array}\right.
\end{aligned}
$$

and the index "(2)" on the last sum indicates that the sum proceeds in steps of two. The second term in (12) is 


$$
A_{1}^{\eta_{2} j_{2}}\left(\rho_{2}, \mathbf{q}_{2}\right)=\frac{e^{-\eta_{2}^{2} / 4 \rho_{2}}}{2^{j_{2}} \sqrt{\pi}} \frac{H_{j_{2}}\left(\eta_{2} / 2 \sqrt{\rho_{2}}\right)}{\rho_{2}^{\left(1+j_{2}\right) / 2}}
$$

As with (57-I)-(61-I)

$$
\begin{aligned}
& \Lambda=\rho_{1}+\rho_{2}, \\
& \Omega=\rho_{1} \rho_{2} y^{2},
\end{aligned}
$$

and

$$
\mathbf{q}_{1}=\mathbf{q}_{2}=\frac{\rho_{2} \mathbf{y}}{\Lambda} .
$$

Then the $\rho$ dependence of the solid spherical harmonic may be factored out,

$$
\mathcal{Y}_{L M}\left(\mathbf{q}_{1}\right)=\left(\frac{\rho_{2}}{\Lambda}\right)^{L} \mathcal{Y}_{L M}(\mathbf{y})
$$

One may change variables from $\rho_{2}$ to

$$
\sigma=\left(\frac{\rho_{2}}{\Lambda}\right)^{1 / 2}
$$

so that for $j_{2}=0$

$$
\frac{d \rho_{2}}{\Lambda^{3 / 2}} A_{1}^{\eta_{2} 0}\left(\rho_{2}, \mathbf{q}_{2}\right)=\frac{2 e^{-\eta_{2}^{2}\left(1-\sigma^{2}\right) / \sigma^{2} 4 \rho_{1}} d \sigma}{\sqrt{\pi} \rho_{1}} .
$$

Finally, the Hermite polynomial may be expanded in $1 / \rho_{1}$,

$$
\begin{aligned}
& \frac{H_{M}\left(\gamma / 2 \sqrt{\rho_{1}}\right)}{\rho_{1}^{(M / 2+1 / 2+1)}} \\
& =\sum_{I=0}^{\left\{\begin{array}{c}
M / 2 \\
(M-1) / 2
\end{array}\right\}} \frac{(-2)^{I}(2 I-1) ! ! M !}{(M-2 I) !(2 I) !} \\
& \times \gamma^{M-2 I} \rho_{1}^{-(M / 2+1 / 2+M / 2-I)-1}
\end{aligned}
$$

with the upper (lower) limit for $M$ even (odd). Defining $N+\frac{1}{2} \equiv M-I+\frac{1}{2}=s^{\prime}+\ell^{\prime}+s+\ell-L+j_{1}-I+\frac{1}{2}$

and

$$
\mu^{2}=\frac{\eta_{2}^{2}\left(1-\sigma^{2}\right)}{\sigma^{2}}+\gamma^{2}
$$

the $\rho_{1}$ integral may be done ${ }^{18}$ giving

$S_{n^{\prime} \ell^{\prime} m^{\prime} \lambda^{\prime}, n \ell m \lambda ; 1}^{\eta_{1} j_{1} ; \eta_{2} 0}(0 ; 0, \mathbf{y})$

$$
\begin{aligned}
= & 4 \pi N_{n^{\prime} \ell^{\prime}} N_{n \ell}\left(\lambda^{\prime} \lambda\right)^{3 / 2} \sum_{s^{\prime}=0}^{n^{\prime}-\ell^{\prime}-1} \frac{(-1)^{s^{\prime}}\left(\lambda^{\prime} / n^{\prime}\right)^{s^{\prime}+\ell^{\prime}}}{\left(n^{\prime}-\ell^{\prime}-1-s^{\prime}\right) !\left(2 \ell^{\prime}+1+s^{\prime}\right) ! s^{\prime} !} \\
& \times \sum_{s=0}^{n-\ell-1} \frac{(-1)^{s}(\lambda / n)^{s+\ell}}{(n-\ell-1-s) !(2 \ell+1+s) ! s !} \sum_{L=L_{m i n}}^{\ell^{\prime}+\ell}(2)(-1)^{m^{\prime}+m} 2^{L}\left(\frac{\left(2 \ell^{\prime}+1\right)(2 \ell+1)(2 L+1)}{4 \pi}\right)^{1 / 2} \\
& \times\left(\begin{array}{ccc}
\ell^{\prime} & \ell & L \\
0 & 0 & 0
\end{array}\right)\left(\begin{array}{ccc}
\ell^{\prime} & \ell & L \\
m^{\prime} m-m^{\prime}-m
\end{array}\right) \sum_{I=0}^{\left\{\begin{array}{c}
M / 2 \\
(M-1) / 2
\end{array}\right\}} \frac{(-2)^{I}(2 I-1) ! ! M !}{(M-2 I) !(2 I) !} \mathcal{Y}_{L, m^{\prime}+m}(y) \frac{2^{M-j_{1}-I}}{\gamma^{N+I+1}} \\
& \times \int_{0}^{1} d \sigma \sigma^{2 L}\left(\frac{\gamma}{\mu}\right)^{2 N+1}\left(\frac{2}{\pi}\right)^{1 / 2}(\mu \sigma y)^{N+1 / 2} K_{N+1 / 2}(\mu \sigma y) .
\end{aligned}
$$

The final integral may be evaluated if $\eta_{2}=0$ so that $\mu=\gamma$. Consider this integral (with argument $z=\gamma R / \nu$ )

$$
J_{L N}(z)=\int_{0}^{1} d \sigma \sigma^{2 L}\left(\frac{2}{\pi}\right)^{1 / 2}(z \sigma)^{N+1 / 2} K_{N+1 / 2}(z \sigma) .
$$

Define a function ${ }^{19}$

$$
f_{N}^{ \pm}(Z)=\left(\frac{2}{\pi}\right)^{1 / 2} e^{Z}(Z)^{ \pm(N+1 / 2)} K_{N+1 / 2}(Z) \text {. }
$$

Then

$$
f_{N}^{+}(Z)=\sum_{k=0}^{N}\left(\begin{array}{c}
N+k \\
2 k
\end{array}\right)(2 k-1) ! ! Z^{N-k}
$$

and

$$
f_{N}^{-}(Z)=\frac{f_{N}^{+}(Z)}{Z^{2 N+1}}
$$

Then ${ }^{20}$

$$
\begin{aligned}
J_{L N}(z)= & \int_{0}^{1} d \sigma \sigma^{2 L} e^{-z \sigma} f_{N}^{+}(z \sigma) \\
= & \sum_{k=0}^{N}\left(\frac{N+k}{2 k}\right)(2 k-1) ! ! \frac{(2 L+N-k) !}{z^{2 L+1}} \\
& \times\left(1-e^{-z} \sum_{u=0}^{2 L+N-k} \frac{z^{u}}{u !}\right) .
\end{aligned}
$$

The spatial integral is thus reduced to a finite series of exponentials. Before performing the time integration, the second term of (11) is needed. But this is a simple overlap integral that may be written down by inspection of (14-I)-(16-I): 


$$
\begin{aligned}
\frac{-1}{R} S_{n^{\prime} \ell^{\prime} m^{\prime} \lambda^{\prime}, n \ell m \lambda}^{01}(0 ; 0)= & \frac{-1}{R} \int d^{3} r u_{n^{\prime} \ell^{\prime} m^{\prime}}^{\lambda^{\prime}}(\mathbf{r}) u_{n \ell m}(\mathbf{r}) \\
= & \frac{-1}{R} N_{n^{\prime} \ell^{\prime}} N_{n \ell}\left(\lambda^{\prime} \lambda\right)^{3 / 2} \sum_{s^{\prime}=0}^{n^{\prime}-\ell^{\prime}-1} \frac{(-1)^{s^{\prime}}\left(2 \lambda^{\prime} / n^{\prime}\right)^{s^{\prime}+\ell^{\prime}}}{\left(n^{\prime}-\ell^{\prime}-1-s^{\prime}\right) !\left(2 \ell^{\prime}+1+s^{\prime}\right) s^{\prime !}} \\
& \times \sum_{s=0}^{n-\ell-1} \frac{(-1)^{s}(2 \lambda / n)^{s+\ell}(-1)^{m^{\prime}}}{(n-\ell-1-s) !(2 \ell+1+s) ! s !} \delta_{\ell \ell^{\prime}} \delta_{-m^{\prime} m} \frac{\left(s^{\prime}+\ell^{\prime}+s+\ell+2\right) !}{(\gamma)^{s^{\prime}+e^{\prime}+s+\ell+3}}
\end{aligned}
$$

\section{TIME INTEGRATION}

Because many of the terms in (33) involve negative powers of $R=\sqrt{B^{2}+v^{2} t^{2}}$, using term-by-term integration of derivatives of the simple integral ${ }^{21}$

$$
\begin{aligned}
\int_{0}^{\infty} e^{-\beta \sqrt{\gamma^{2}+t^{2}}} & \cos b t d t \\
& =\frac{\beta \gamma}{\sqrt{\beta^{2}+b^{2}}} K_{1}\left(\gamma \sqrt{\beta^{2}+b^{2}}\right)
\end{aligned}
$$

will not suffice. This difficulty is surmounted by a particular grouping of terms. It is shown in the Appendix that the simple sum in the last term of (33) may be rewritten in terms of the function $f^{ \pm}(30)$,

$$
S_{L}(z)=\sum_{u=0}^{2 L-1} \frac{z^{u-2 L-1}}{u !}=\frac{1}{(2 L-1) ! !} \sum_{j=1}^{L} \frac{f_{j}^{-}(z)}{[2(L-j)] ! !}
$$

and

$$
\begin{aligned}
T_{L, g}(z) & =\sum_{u=2 L}^{2 L+g+1} \frac{z^{u-2 L-1}}{u !} \\
& =\frac{f_{0}^{-}(z)}{(2 L) !}+\sum_{h=0}^{g} \sum_{j=h}^{g} \frac{a_{j}^{h} f_{h}^{+}(z)}{(2 L+1+j) !}
\end{aligned}
$$

where the $a_{j}^{h}$ are given by (A11). Then the time integral may be evaluated using ${ }^{22}$

$$
\int_{0}^{\infty}\left(t^{2}+b^{2}\right)^{(1 / 2) \nu} K_{ \pm \nu}\left(a \sqrt{t^{2}+b^{2}}\right) t \sin (c t) d t=\left(\frac{\pi}{2}\right)^{1 / 2} a^{\nu} b^{\nu+3 / 2} c\left(a^{2}+c^{2}\right)^{-(1 / 2) \nu-3 / 4} K_{-\nu-3 / 2}\left(b \sqrt{a^{2}+c^{2}}\right)
$$

and

$$
\begin{aligned}
\int_{0}^{\infty}\left(t^{2}+b^{2}\right)^{\mp(1 / 2) \nu} K_{\nu}\left(a \sqrt{t^{2}+b^{2}}\right) \cos (c t) d t= & \left(\frac{\pi}{2}\right)^{1 / 2} a^{\mp \nu} b^{1 / 2 \mp \nu}\left(a^{2}+c^{2}\right)^{ \pm(1 / 2) \nu-1 / 4} \\
& \times K_{ \pm \nu-1 / 2}\left(b \sqrt{a^{2}+c^{2}}\right)(\operatorname{Re} a>0, \operatorname{Re} b>0, c>0)
\end{aligned}
$$

where $K_{\nu}$ is the modified Bessel function of the second kind.

In order to use (39) one must first expand the solid harmonic,

$$
\begin{aligned}
\mathcal{Y}_{L M}\left(\frac{B}{\nu} \hat{\mathbf{i}}+\frac{v}{\nu} t \hat{\mathbf{k}}\right) e^{i \omega t}= & \frac{1}{\nu^{L}} \mathcal{Y}_{L M}\left(B \hat{\mathbf{i}}+v\left(-i \frac{\partial}{\partial \omega}\right) \hat{\mathbf{k}}\right) e^{i \omega t} \\
= & \frac{1}{\nu^{L}}\left(\frac{2 L+1}{4 \pi}(L+M) !(L-M) !\right)^{1 / 2} \\
& \times \sum_{q} \frac{(-B)^{q+M}(B)^{q}(v)^{L-M-2 q}}{2^{2 q+M}(q+M) ! q !(L-M-2 q) !}\left(-i \frac{\partial}{\partial \omega}\right)^{L-M-2 q} e^{i \omega t},
\end{aligned}
$$

where the sum runs over all values of $q$ for which the factorials are non-negative.

The final result for (11) is ${ }^{23}$ 


$$
\begin{aligned}
& \mathcal{A}_{n^{\prime} \ell^{\prime}-m^{\prime}, n \ell m}^{\lambda^{\prime}} \stackrel{\lambda}{\lambda}(\omega, B, \nu, Z) \\
& =(-1)^{-m^{\prime}} \frac{Z_{p} Z}{v} N_{n^{\prime} \ell^{\prime}} N_{n \ell}\left(\lambda^{\prime} \lambda\right)^{3 / 2} \sum_{s^{\prime}=0}^{n^{\prime}-\ell^{\prime}-1} \frac{(-1)^{s^{\prime}}\left(\lambda^{\prime} / n^{\prime}\right)^{s^{\prime}+\ell^{\prime}}}{\left(n^{\prime}-\ell^{\prime}-1-s^{\prime}\right) !\left(2 \ell^{\prime}+1+s^{\prime}\right) ! s !} \sum_{s=0}^{n-\ell-1} \frac{(-1)^{s}(\lambda / n)^{s+\ell}}{(n-\ell-1-s) !(2 \ell+1+s) ! s !} \\
& \times\left\{-\delta_{\ell \ell^{\prime}} \delta_{m m^{\prime}} 2^{s^{\prime}+\ell^{\prime}+s+\ell} \frac{\left(s^{\prime}+\ell^{\prime}+s+\ell+2\right) !}{\gamma^{s^{\prime}+\ell^{\prime}+s+\ell+3}} K_{0}(\omega B / v)\right. \\
& +4 \pi \sum_{L=L_{\min }}^{\ell^{\prime}+\ell}(2) 2^{L}\left(\frac{\left(2 \ell^{\prime}+1\right)(2 \ell+1)(2 L+1)}{4 \pi}\right)^{1 / 2}\left(\begin{array}{ccc}
\ell^{\prime} & \ell L \\
0 & 0 & 0
\end{array}\right)\left(\begin{array}{ccc}
\ell^{\prime} & \ell & L \\
m^{\prime} & m & -m^{\prime}-m
\end{array}\right) \\
& \times \sum_{I=0}^{\left\{\begin{array}{c}
M / 2 \\
(M-1) / 2
\end{array}\right\}} \frac{(-1)^{I}(2 I-1) ! ! M !}{(M-2 I) !(2 I) !} \frac{1}{\gamma^{M+1}} 2^{M-1} \frac{1}{\nu^{L+1}}\left(\frac{2 L+1}{4 \pi}\left(L+m^{\prime}+m\right) !\left(L-m^{\prime}-m\right) !\right)^{1 / 2} \\
& \times \sum_{q} \frac{(-1)^{q} B^{2 q+m^{\prime} m} v^{L-m^{\prime}-m-2 q}}{2^{2 q+m^{\prime}+m}\left(q+m^{\prime}+m\right) ! q !\left(L-m^{\prime}-m-2 q\right) !}\left(-i \frac{\partial}{\partial \omega}\right)^{L-m^{\prime}-m-2 q} 2 \\
& \times \sum_{k=0}^{N}\left(\begin{array}{c}
N+k \\
2 k
\end{array}\right)(2 k-1) ! !(2 L+N-k) ! \\
& \times\left[\left(\frac{\omega}{2 B v}\right)^{L} \frac{\nu^{2 L+1}}{\gamma^{2 L+1}} \frac{\sqrt{\pi}}{\Gamma\left(\frac{1}{2}+L\right)} K_{L}(B \omega / v)\right. \\
& -\frac{1}{(2 L-1) ! !} \sum_{j=1}^{L} \frac{1}{[2(L-j)] ! !} \frac{\nu^{2 j+1}}{\gamma^{2 j+1}}\left(\frac{A}{B}\right)^{j} K_{j}(B A)-\frac{1}{(2 L) !} \frac{\nu}{\gamma} K_{0}(B A) \\
& \left.\left.-\sum_{h=0}^{N-k-1} \sum_{j=h}^{N-k-1} \frac{a_{j}^{h}}{(2 L+1+j) !} \frac{\gamma^{2 h+1}}{\nu^{2 h+1}}\left(\frac{B}{A}\right)^{h+1} K_{h+1}(B A)\right]\right\}
\end{aligned}
$$

where

$$
A=\left(\frac{\gamma^{2}}{\nu^{2}}+\frac{\omega^{2}}{v^{2}}\right)^{1 / 2}
$$

For example,

$$
\begin{aligned}
& \mathcal{A}_{200,100}^{\lambda^{\prime}{ }_{0}^{\lambda}}\left(\omega, B, \nu, Z_{e}\right)=\frac{Z_{P} Z_{e}}{v} 2^{3 / 2} \frac{\left(\lambda^{\prime} \lambda\right)^{3 / 2}}{\gamma^{3}}\left[-2\left(\frac{\lambda-\lambda^{\prime}}{\gamma}\right) K_{0}(\alpha B)\right. \\
& +\frac{\left(\lambda-\lambda^{\prime}\right)}{\gamma}\left(2\left[K_{0}(\alpha B)-K_{0}(B A)\right]-\frac{\gamma^{2}}{\nu^{2}} \frac{B}{A} K_{1}(B A)\right) \\
& \left.+\frac{\lambda^{\prime}}{2} \frac{\gamma^{3}}{\nu^{4}} \frac{B^{2}}{A^{2}} K_{2}(B A)\right]
\end{aligned}
$$

where

$$
\alpha=\frac{\omega}{v} .
$$

Note that the first term in (43), arising from monopole term in (8) [the Kronecker $\delta$ term in (41)] exactly can- cels the second term of (43). Thus it is seen that the proper decomposition of the Hamiltonian to give planewave asymptotic states (6) removes the term in the transition amplitude that diverges at zero energy difference $\omega$. This is an important consideration for the second-order 
contributions. If there is no change in screening for the $1 \mathrm{~s} \rightarrow 2 \mathrm{~s}$ transitions, then $\lambda=\lambda^{\prime}$ and the monopole terms drop out anyway. However, elastic scattering amplitudes do not have a $\lambda-\lambda^{\prime}$ coefficient so the cancellation from the overlap integral (the $\delta_{\ell \ell^{\prime}} \delta_{m m^{\prime}}$ term) is critical.

Equation (43) is identical to the expression derived us- ing an entirely different integration scheme ${ }^{13}$ (for $\nu=1$ ). Setting $Z_{P}=\lambda^{\prime}=\lambda=\nu=-Z_{e}=1$ and multiplying by the factor $(-i)$ in (10) gives the transition amplitude found by Van Den Bos and De Heer. ${ }^{1}$

For transitions that change $\ell$ or $m$, the Kronecker $\delta$ term does not contribute. However, a typical result

$$
\begin{aligned}
& \mathcal{A}_{21-1,211}^{\lambda^{\prime}{ }^{\lambda}}\left(\omega, B, \nu, Z_{e}\right)=-\frac{Z_{P} Z_{e}}{v}\left(\lambda^{\prime} \lambda\right)^{5 / 2} 3 \frac{\nu^{2}}{\gamma^{7}}\left[2 \alpha^{2} K_{2}(\alpha B)-\left(2 A^{2}+\frac{\gamma^{4}}{4 \nu^{4}} B^{2}\right) K_{0}(B A)\right. \\
& \left.-\left(\frac{\gamma^{2}}{\nu^{2}} A B+4 \frac{A}{B}+\frac{\gamma^{6} B^{3}}{\left(\nu^{6} 3\right)\left(2^{3} A\right)}\right) K_{1}(B A)\right]
\end{aligned}
$$

is seen to be finite as $\alpha \rightarrow 0$. Again, this is the result found previously by alternate methods. ${ }^{13}$

Finally, consider

$$
\begin{aligned}
& \mathcal{A}_{310,100}^{\lambda^{\prime} \underset{1}{\lambda}}\left(\omega, B, \nu, Z_{e}\right)=i \frac{Z_{P} Z_{e}}{v} \frac{\lambda^{\prime 5 / 2} \lambda^{3 / 2}}{\gamma^{5}} \nu \frac{2^{4}}{3^{3}} \sqrt{2}\left\{2\left(4-\frac{10 \lambda^{\prime}}{3 \gamma}\right)\left[\alpha K_{0}(\alpha B)-\alpha K_{0}(A B)\right]\right. \\
& +\left[\frac{1}{6} \frac{\lambda^{\prime} \gamma^{5}}{\nu^{6}} \frac{B^{3}}{A^{3}} \alpha-\frac{\gamma^{2}}{\nu^{2}}\left(4-\frac{10 \lambda^{\prime}}{3 \gamma}\right) \frac{B}{A} \alpha\right] K_{1}(A B) \\
& \left.+\left[\frac{2}{3} \frac{\lambda^{\prime} \gamma^{5}}{\nu^{6}} \frac{B^{2}}{A^{4}} \alpha-\left(1-\frac{5 \lambda^{\prime}}{2 \gamma}\right) \frac{\gamma^{4}}{\nu^{4}} \frac{B^{2}}{A^{2}} \alpha\right] K_{2}(A B)\right\} \text {. }
\end{aligned}
$$

Setting $\lambda=\lambda^{\prime}=Z_{P}=\nu=-Z_{e}=1,(10)$ gives

$$
\begin{aligned}
a_{310,100}^{1}(\omega, B)=-\frac{3}{2^{6}} 3 \sqrt{2}[ & \alpha K_{0}(\alpha B)-\alpha K_{0}(A B)+\left(\frac{2^{9}}{3^{7}} \frac{B^{3}}{A^{3}} \alpha-\frac{2^{3}}{3^{2}} \frac{B}{A} \alpha\right) K_{1}(A B) \\
& \left.+\left(\frac{2^{11}}{3^{7}} \frac{B^{2}}{A^{4}} \alpha-\frac{2^{5}}{3^{4}} \frac{B^{2}}{A^{2}} \alpha\right) K_{2}(A B)\right] .
\end{aligned}
$$

This is $(-i)^{-1}$ times the result of Van Den Bos and De Heer. ${ }^{1}$ This phase difference of $(-i)^{-\left(\ell^{\prime}+\ell\right)}$ is consistent with the results calculated on the $n=1$ and 2 manifolds using both independent methods. ${ }^{13}$ This phase may be accounted for by noting that Van Den Bos and De Heer cite Podolsky and Pauling ${ }^{24}$ as the source of their momentum wave function. Weniger ${ }^{25}$ has shown that those wave functions are missing a factor of $(-i)^{\ell^{\prime}+\ell}$. The present results agree with the calculation using the technique of Van Den Boss and De Heer, but with the correct momentum wave functions of Weniger. It should be noted that the incorrect phase of Van Den Bos and De Heer does not affect the first-order cross sections used in comparison with experiment. ${ }^{2-5}$ But the correct phase is critical when considering a second-order calculation in which the principal-value part of the second-order amplitude interferes with the first-order amplitude.

\section{CONCLUSIONS}

A closed-form analytic expression has been found for the first-order amplitude for the general state-to-state transition of a one-electron atom due to projectile impact. Thus a first-order theory is now available for any transition, be it ground state to Rydberg state or between excited states. Because the present result is analytic, the second-order amplitude contains at most a one-dimensional principal-value integral of a product of two of these first-order amplitudes. ${ }^{13}$ Likewise a third- or fourth-order result for excitation may be calculationably feasible since these amplitudes would involve at most two or three integrals, respectively.

\section{ACKNOWLEDGMENTS}

This work was supported by the Division of Chemical Sciences, Office of Energy Research, U.S. Department of Energy.

\section{APPENDIX}

It is first desired to prove (36) that

$$
S_{L}^{\prime}(z)=\sum_{u=0}^{2 L-1} \frac{z^{u-2 L-1}}{u !}
$$


equals

$$
S_{L}=\frac{1}{(2 L-1) ! !} \sum_{j=1}^{L} \frac{f_{j}^{-}(z)}{[2(L-j)] ! !}
$$

where (30)

$$
\begin{aligned}
f_{j}^{-}(z) & =\left(\frac{2}{\pi}\right)^{1 / 2} e^{z}(z)^{-(j+1 / 2)} K_{j+1 / 2}(z) \\
& =\sum_{k=0}^{j}\left(\begin{array}{c}
j+k \\
2 k
\end{array}\right)(2 k-1) ! ! \frac{1}{z^{N+k+1}} .
\end{aligned}
$$

The proof proceeds by induction. First it is seen that

$$
S_{1}=\left(\frac{1}{z^{2}}+\frac{1}{z^{3}}\right)=S_{1}^{\prime}
$$

and

$$
\begin{aligned}
S_{2} & =\frac{1}{3}\left[\frac{1}{2}\left(\frac{1}{z^{2}}+\frac{1}{z^{3}}\right)+\left(\frac{1}{z^{3}}+\frac{3}{z^{4}}+\frac{3}{z^{5}}\right)\right] \\
& =\frac{1}{z^{5}}+\frac{1}{z^{4}}+\frac{1}{z^{3}} \frac{(1+2)}{(3)(2)}+\frac{1}{z^{2} 3 !}=S_{2}^{\prime} .
\end{aligned}
$$

Now given that for some $\mathrm{L}$ one has $S_{L}=S_{L}^{\prime}$, let it be shown that $S_{L+1}=S_{L+1}^{\prime}$.

First note that

$$
\begin{aligned}
S_{L+1}^{\prime} & =\sum_{u=0}^{2 L+1} \frac{z^{u-2 L-2-1}}{u !} \\
& =\frac{1}{z^{2}} \sum_{u=0}^{2 L-1} \frac{z^{2-2 L-1}}{u !}+\sum_{u=2 L}^{2 L+1} \frac{z^{u-2 L-3}}{u !} \\
& =\frac{1}{z^{2}} S_{L}^{\prime}+\sum_{u=0}^{1} \frac{z^{u-3}}{(v+2 L) !} .
\end{aligned}
$$

Second, using recursion relations ${ }^{26}$ for $K_{\nu}(z)$ it may be shown that

$$
f_{n+1}^{-}(z)=\frac{(2 n+1) f_{n}^{-}(z)+f_{n-1}^{-}(z)}{z^{2}} .
$$

$$
\begin{aligned}
& S_{L+1}(z)= \frac{1}{(2 L+1) ! !}\left(\frac{f_{1}^{-}(z)}{(2 L) ! !}+\sum_{j=2}^{L+1} \frac{f_{j}^{-}(z)}{[2(L+1-j)] ! !}\right) \\
&= \frac{f_{1}^{-}(z)}{(2 L+1) !}+\frac{1}{(2 L+1)(2 L-1) ! !} \sum_{j=2}^{L+1} \frac{(2 j-1) f_{j-1}^{-}(z)+f_{j-2}^{-}(z)}{z^{2}[2(L+1-j)] ! !} \\
&= \frac{f_{1}^{-}(z)}{(2 L+1) !}+\frac{1}{z^{2}(2 L-1) ! !} \sum_{j=2}^{L+1} \frac{f_{j-1}^{-}(z)}{\{2[L-(j-1)]\} ! !} \\
&+\frac{1}{z^{2}(2 L+1) ! !}\left(\sum_{j=2}^{L+1} \frac{[2 j-1-(2 L+1)] f_{j-1}^{-}(z)}{[2(L+1-j)] ! !}+\sum_{j=2}^{L+1} \frac{f_{(j-1)-1}^{-}(z)}{\{2[L-(j-1)]\} ! !}\right) \\
&= \frac{f_{1}^{-}(z)}{(2 L+1) !}+\frac{1}{z^{2}(2 L-1) ! !} \sum_{k=1}^{L} \frac{f_{k}^{-}(z)}{[2(L-k)] ! !} \\
&+\frac{1}{z^{2}(2 L+1) ! !}\left(\sum_{j=2}^{L+1} \frac{2(j-L-1)}{2(L-j+1)} \frac{f_{j-1}^{-}(z)}{[2(L-j)] ! !}+\sum_{k=1}^{L} \frac{f_{k-1}^{-}(z)}{[2(L-k)] ! !}\right) \\
&=\left.\frac{f_{1}^{-}(z)}{(2 L+1) !}+\frac{1}{z^{2}} S_{L}+\frac{1}{z^{2}(2 L+1) ! !} \frac{1}{f_{0}^{-}(z)}\right) \\
&= \frac{1}{z^{2}} S_{L}^{\prime}+\frac{1}{(2 L+1) !}\left[\left(\frac{1}{z^{2}}+\frac{1}{z^{3}}\right)+\frac{2 L}{z^{3}}\right]=S_{L+1}^{\prime}(z) \\
&(2 L-2) ! !
\end{aligned}
$$

Thus it has been shown that for any $\mathrm{L}$ one has $S_{L}=S_{L}^{\prime}$.

For powers of $z$ greater than $z^{-2}$, that is

$$
T_{L, g}(z)=\sum_{u=2 L}^{2 L+g+1} \frac{z^{u-2 L-1}}{u !}=\frac{1}{(2 L) ! z}+\sum_{i=0}^{g} \frac{z^{i}}{(2 L+1+i) !}
$$


one may simply note that the set $f_{n}^{+}(z)$ spans the space of polynomials. In fact

$$
z^{i}=\sum_{k=0}^{i} a_{i}^{i-k} f_{i-k}^{+}(z)
$$

where the $a$ 's are given by recursion,

$$
\begin{aligned}
& a_{i}^{i} \equiv 1 \\
& a_{i}^{i-1}=-\left(\begin{array}{c}
i+1 \\
2
\end{array}\right) \\
& a_{i}^{i-k}=\sum_{m=0}^{k} a_{i}^{i-m}\left(\begin{array}{c}
i+k-2 m \\
2 k-2 m
\end{array}\right)(2 k-2 m-1) ! ! \\
& a_{i}^{0} \equiv 0
\end{aligned}
$$

Combining common factors of $f_{h}^{+}(z)$ gives

$$
T_{L, g}(z)=\frac{f_{0}^{-}(z)}{(2 L) !}+\sum_{h=0}^{g} \sum_{j=h}^{g} \frac{a_{j}^{h} f_{h}^{+}(z)}{(2 L+1+j) !} .
$$

${ }^{1}$ J. Van Den Bos and F.J. De Heer, Physica 34, 333 (1967); 40, 161(E) (1968).

${ }^{2}$ F. Hopkins et al., Phys. Rev. A 13, 74 (1976).

${ }^{3}$ J.T. Park, J.E. Alday, J.M. George, and J.L. Peacher, Phys. Rev. A 14, 608 (1976).

${ }^{4}$ F. Hopkins and A. Little, Phys. Rev. A 14, 1634 (1976).

${ }^{5}$ K. Reyman, K.-H. Schartner, B. Sommer, and E. Träbert, Phys. Rev. A 38, 2290 (1988).

${ }^{6}$ L. Kocbach, J. Phys. B 9, 2269 (1976).

${ }^{7}$ U. Singer and J.C. DeHaes, Nucl. Instrum. 202, 227 (1982).

${ }^{8}$ T.L. McAbee, Nucl. Instrum. 214, 89 (1983).

${ }^{9}$ J.O.P. Pedersen and P. Hvelplund, Phys. Rev. Lett. 62, 2373 (1989).

${ }^{10}$ J. P. Giese, M. Schulz, J. K. Swensen, H. Schoene, M. Beahenn, S. L. Varghese, C. R. Vane, P. F. Dittner, S. M. Shafroth, and S. Datz, Phys. Rev. A 42, 1231 (1990).

${ }^{11}$ J.O.P. Pedersen and F. Folkman, J. Phys. B 23, 441 (1990).

${ }^{12}$ J.H. McGuire, D.J. Land, J.G. Brennan, and G. Basbas, Phys. Rev. A 19, 2180 (1979).

${ }^{13}$ J.C. Straton and J.H. McGuire (unpublished).

${ }^{14}$ R. McCarroll and A. Salin, C. R. Acad. Sci. 263, 329 (1966).

${ }^{15}$ M.R.C. McDowell and J.P. Coleman, Introduction to the Theory of Ion-Atomic Collisions (North-Holland, Amsterdam, 1970), Appendix 4.1.
${ }^{16}$ C.G.J. Jacobi, J. Math. 36, 115 (1843).

${ }^{17}$ J.C. Straton, Phys. Rev. A 41, 71 (1990).

${ }^{18}$ I.S. Gradshteyn and I.M. Ryzhik, in Table of Integrals, Series, and Products, edited by A. Jeffrey (Academic, New York, 1980), p. 340, No. 3.471(9).

${ }^{19}$ This function may be related to generalized Laguerre polynomials by

$$
f_{N}^{ \pm}(z)=\left[N !(-1)^{N} / 2^{N}\right]\left\{\begin{array}{c}
1 \\
z^{-(2 N+1)}
\end{array}\right\} L_{N}^{-(2 N+1)}(2 z) .
$$

See A.P. Prudnikov, Yu. A. Brychkov, and O.I. Marichev, Integrals and Series (Gordon and Breach, New York, 1986), Vol. 2, p. 730 .

${ }^{20}$ I.S. Gradshteyn and I.M. Ryzhik, Ref. 18, p. 310, No. 3.351(1).

${ }^{21}$ I. S. Gradshteyn and I. M. Ryzhik, Ref. 18, p. 482, No. 3.914 .

${ }^{22}$ I. S. Gradshteyn and I. M. Ryzhik, Ref. 18, p. 756, No. $6.726(3,4)$.

${ }^{23}$ I. S. Gradshteyn and I. M. Ryzhik, Ref. 18 , p. 426 , No. $3.771(2,5)$.

${ }^{24}$ B. Podolsky and L. Pauling, Phys. Rev. 34, 109 (1929).

${ }^{25}$ E.J. Weniger, J. Math. Phys. 26, 276 (1985), Eq. 4.30.

${ }^{26}$ I.S. Gradshteyn and I.M. Ryzhik, Ref. 18, p. 970, No. $8.486(10)$. 